Ann. Génét. Sél. anim., I972, 4 (I), 79-85.

\title{
UTILISATION CONSCIENTE ET INCONSCIENTE DE CERTAINS FACTEURS MENDÉLIENS DANS LA SÉLECTION DES GROSSES ESPECES DOMESTIQUES ( $\left.{ }^{1}\right)$
}

\author{
J.-J. LAUVERGNE \\ Laboratoire de Génétique factorielle, \\ Centre national de Recherches zootechniques, I. N. R. A., \\ 78 - Jouy-en-Josas \\ RÉSUMÉ
}

De récentes études ont montré que la plupart des mutants en ségrégation ordinaire dans les espèces domestiques ne présentent pas des qualités telles que le sélectionneur doive leur faire un sort individuel. Aussi se justifie dans une certaine mesure l'importance prise en sélection par la génétique quantitative.

Il existe cependant, une catégorie de gènes à effets pléiotropiques ambigus, à la fois favorables et défavorables, qui peuvent améliorer grandement la qualité des animaux en exploitation. Dans le passé, à l'insu des éleveurs, ils ont déjà été utilisés et cela explique certaines différences entre races domestiques et sauvages, bien avant que n'intervienne la sélection rationnelle. Actuellement, à la suite de la réussite de la sélection de la Poule naine, ils suscitent un certain intérêt chez les grosses espèces de Mammifères, comme en témoigne l'abondance des travaux menés sur le caractère d'hypertrophie musculaire des Bovins (culard).

La règle commune d'emploi de ces gènes est de minimiser leurs défauts en exaltant leurs qualités, ce qui peut conduire à des utilisations variées en souche pure ou en croisements.

De tels " bons gènes " sont toutefois assez rares : aussi, pour le moment, on est loin de pouvoir compter uniquement sur eux pour établir des programmes de sélection. Cependant, avec les progrès de "l'ingénieurie génétique " qui nous promet la création à volonté de nouveaux mutants, il se peut que la situation se renverse.

\section{INTRODUCTION}

Après la redécouverte des Lois de Mendel, au début du siècle on notait, en Amérique un très fort engouement pour les études factorielles, même chez les gros animaux domestiques LUSH, (I956).

(') Rapport présenté à la Réunion annuelle de la Fédération européenne de Zootechnie, Commission de Génétique, Versailles, France, le I7 juillet I97I. 
Toutefois, un quart de siècle plus tard, si quelques-unes de ces études se poursuivaient encore on n'espérait plus en tirer un avantage quelconque en sélection.

Toujours en Amérique est alors née la doctrine nouvelle d'une sélection basée sur la génétique quantitative (début des années trente). Les résultats obtenus par cette science appliquée furent alors si concluants que, depuis lors, les sélectionneurs d'animaux domestiques sont essentiellement des généticiens biométriciens.

Cependant, un instant ralenties, les études mendéliennes des animaux domestiques reprenaient dans les années quarante avec le développement de l'immunogénétique, complétées un peu plus tard par les études de polymorphisme des protéines libres du sang et du lait et des enzymes, grâce à l'électrophorèse.

Devant cette affluence de nouveaux loci et allèles les auteurs ont commencé, vers le milieu des années cinquante, à en étudier l'éventuelle influence sur la productivité. Par ailleurs, d'autres chercheurs entreprenaient des études identiques sur des loci plus classiquement identifiables (couleur, variations morphologiques diverses, anomalies).

Certains de ces travaux ont déjà débouché sur des progrès sélectifs comparables à ceux que procurent les plans de sélection basés sur la génétique quantitative. Devant ces résultats déjà intéressants le temps semble venu de s'interroger sur l'avenir de telles recherches mendéliennes.

Tout d'abord les anciens éleveurs n'ont-ils pas, à leur insu, utilisé de tels moyens factoriels et n'y a-t-il pas encore quelque chose à recueillir de leur héritage?

En second lieu il est intéressant de voir comment ces nouvelles applications peuvent s'insérer dans les plans de sélection existants.

En troisième lieu si, déjà, l'utilisation de gènes connus sur leur seul comportement extérieur donne des résultats, ne doit-on pas s'attendre à d'autres avantages lorsque, avec les progrès de la génétique moléculaire, on connaîtra mieux le matériel génique?

\section{BILAN DES RÉCENTS TRAVAUX}

\section{CONSACRÉS AUX INFLUENCES GÉNÉTIQUES INDIVIDUELLES}

\section{SUR LA PRODUC'TIVITÉ DES ANIMAUX DOMESTIQUES}

Les récents travaux sur l'influence de divers allèles sur la productivité ont eu deux orientations principales. La première porte uniquement sur les groupes sanguins et les polymorphismes, surtout dans l'espèce bovine, la seconde sur les gènes à effet visible, puis aussi sur les polymorphismes, chez la Poule domestique.

Les chercheurs qui ont choisi la première orientation étaient, au début tout au moins, anglo-saxons ou nordiques. On peut citer les noms de ROBERTSON, RENDEL, NEIMANN-SÖRENSEN.

Les auteurs de travaux sur la Poule sont originaires de divers pays mais surtout des États-Unis, HuTT, BERnIER... et de France où ces études ont été un des soucis constants de MÉRat qui, pour les applications pratiques, a collaboré avec CochEZ de la Station de Sélection avicole du Magneraud.

Ces travaux ont été résumés à de multiples reprises, signalons les revues de Robertson (I96I, I966, I970), de Buschmann et Schmid (r968) pour les gros Mam- 
mifères, de Nordskog (I964), MÉrat et Perramon (Ig68), MÉrat (I970) chez la Poule.

Les études sur les gros Mammifères corroborent les études plus étendues et plus précises faites chez la Poule. Les gènes analysés - qui sont pour la plupart des allèliques ségréguent naturellement dans toutes les races ou bien des gènes d'agrément retenus dans telle ou telle race — ont, en général, une petite influence sur tel ou tel caractère de production. Cette influence, qui souvent s'exerce en overdominance (ce qui explique le maintien de ségrégation) n'est généralement pas utilisable par le sélectionneur $\left({ }^{1}\right)$.

En fait les utilisations pratiques dont nous aurons fait mention dans 1'introduction de cet article se sont basées sur des gènes à action plus complexe dont l'intérêt n'est d'ailleurs pas apparu immédiatement.

Par exemple, au cours de ses recherches systématiques, MÉRAT a découvert (ou redécouvert) un gène de nanisme lié au sexe qui, à première vue, exerçait un effet dépressif sur la croissance, la ponte, le poids des œufs. Une étude plus précise a cependant montré que l'effet dépressif était inférieur à l'économie réalisée sur l'entretien et la nourriture de l'animal. D'où l'idée première de la confier à CochEZ, au Magneraud, pour l'introduire dans une souche de pondeuses.

Finalement le gain économique était encore plus considérable si on l'introduisait dans une souche de mères de poussins de chair (allant jusqu'à un gain de 20 p. Ioo pour la production de poussin d'un jour) ce qui a été fait et a donné la souche Vedette I. N.R. A. (MÉrAT, I972).

Chez les Bovins, à un stade moins avancé de commercialisation, il existe actuellement un programme consacré au gène d'hypertrophie musculaire (culard, Doppellender, double-muscle) qui améliore spectaculairement les qualités de carcasse tout en présentant de multiples défauts. A cet effet, en France, l'Institut national de la Recherche agronomique a même acquis et aménagé un domaine expérimental.

Ainsi apparaît une catégorie de gènes à effets pléitropiques ambigus sur la productivité. Dans un programme de sélection quantitative ils seront, la plupart du temps, éliminés et leur emploi doit être conçu en faisant la balance de leurs avantages et inconvénients. On peut penser cependant que, par le passé, certains éleveurs aient pu les utiliser avec les risques que l'on entrevoit lorsque l'ensemble de leur comportement n'est pas élucidé.

Effectivement, dans l'espèce bovine, où nous avons déjà tiré l'exemple du culard, ils sont légion et, jusqu'à présent, leur influence a été plutôt dépressive. Leur fréquence semble avoir été augmentée en se basant uniquement sur des considérations esthétiques fort peu en rapport avec la productivité. C'est ainsi que les éleveurs anglais ou américains, obnubilés par la recherche d'un animal " compact ", ont utilisé successivement un gène comme le "bulldog du Dexter " à effet dominant mais létal récessif, puis le "Snorter-dwarf " également fort peu viable (cf. LAUVERGNE, I968; Catalogue des anomalies).

Le maintien du gène rouan dans la race Shorthorn (RENDEL, I952) ou de Haute et Moyenne Belgique (HANset, I965) et celui du blanc dans la race Suédoise des Montagnes (LAUVERGNE, I970) relèvent également de considérations fort peu économiques quand on sait que ces 2 facteurs exercent un effet néfaste sur la fertilité.

(1) Nous omettons ici volontairement l'utilisation des déterminations géniques pour le contrôle de la parenté, outil très précieux de la sélection, mais il s'agit d'une utilisation indirecte. De même l'énorme intérêt de l'analyse des séquences aminées pour l'Évolution sort de notre champ de vision. 
On a peu de données concernant l'action zootechnique des mutants d'absence de cornes chez les bovins mais, chez la chèvre d'origine alpine, on sait que le caractère motte, longtemps pris comme standard dans plusieurs races, est régi par un gène spécialement défavorable à la reproduction puisqu'il stérilise tous les animaux d'un certain génotype (LAUVERGNE, I969).

Plus fructueuse semble avoir été 1'adoption déjà ancienne du caractère " mérinos " chez le mouton qui, selon nous, résulterait d'une unique mutation. On s'expliquerait mal en effet comment un tel faisceau d'influences surla pousse de la laine, le type de fibre, la saison sexuelle, la fertilité aient pu, à travers tant de générations, se transmettre en bloc. Avec le "mérinos " le profit économique a été certain et le nom de toison d'or n'est pas usurpé, à côté de pertes relativement minimes surtout vis-à-vis de races non sélectionnées.

Si l'on pouvait mieux marquer les gènes et si l'on se préoccupait d'étudier plus systématiquement les influences possibles on découvrirait certainement de nombreux autres exemples. Les animaux domestiques présentent vis-à-vis de leurs congénères sauvages un certain nombre d'avantages, qui ont automatiquement leur contrepartie. Ainsi, par exemple les gènes conditionnant l'utilisation des sucres et des graisses avec les symptômes diabétiques qui apparaissent dans les souches les mieux adaptées aux milieux arides, dès lors qu'on les nourrit ad libitum. C'est certainement une des voies par lesquelles la rusticité peut s'opposer à la productivité. Cet exemple mérite d'être médité lorsque l'on veut, pour des raisons économiques, rester dans des conditions d'élevage extensif.

\section{LES BASES D'UN EMPLOI RATIONNEL, DES FAC'TEURS MENDÉLIENS}

\section{UTILISABLES INDIVIDUELLEMENT}

\section{I. - Portrait-robot du mutant utile}

Les mutants qui méritent un traitement séparé pour leur utilisation en sélection se classent à première vue dans la catégorie des gènes délétères, du moins vis-à-vis de certains caractères de production et de reproduction mais ils ont une action favorable sur un critère très intéressant : pour le culard ce sera les qualités de carcasse, pour la Poule naine l'économie dans l'entretien d'une souche mère de poussins. Pour d'autres mutants ce pourra être la résistance à telle maladie, 1'absence de cornes, la résistance à la sécheresse...

Dans cette perspective on peut imaginer la recherche systématique, par exemple chez les Bovins, non pas d'un gène réducteur esthétique du format mais plutôt d'un gène de nanisme conservant à la femelle l'essentiel de ses qualités de fertilité et de fécondité avec des mises-bas faciles. On obtiendrait alors bien plus rapidement que par une sélection quantitative des souches de femelles " moules à veaux " dont HILI' (I97I) repousse la création pour des raisons de coût excessif. La chance de trouver de tels mutants dépend évidemment de la taille de la population contrôlée qui doit être de plusieurs millions d'individus si on veut avoir des chances d'aboutir à brève échéance.

Si l'on se propose d'étudier en détail le comportement des nouveaux mutants 
il sera beaucoup plus facile de faire sur les effectifs expérimentaux, forcément réduits. des mesures que l'on hésite encore à réaliser au niveau des populations en cours de sélection. Par exemple, on pourrait étudier l'appétit, le coefficient de transformation, le caractère... dont l'incidence économique est également importante, bien qu'encore négligée la plupart du temps.

\section{2. - Emploi des mutants individuels}

A propos de la Poule naine on a vu les hésitations qui avaient accompagné son introduction dans les souches commerciales. Le caractère ambigu de tels mutants rend en effet délicat leur emploi. Il s'agit de minimiser leurs défauts en exaltant leurs qualités.

A cet égard l'exemple du culard est tout à fait probant lui aussi. Tout à tour on l'a vu porté aux nues par exemple dans la race piemontese della "Coscia ", à cause de ses qualités de carcasses ou sévèrement pourchassé à cause de son effet dépressif sur la production laitière (en race pie noire allemande par ex.) (LAUVERGNe et al., I968.)

Dans les races à viande comme le Charolais ou la race de Haute et Moyenne Belgique, sa présence est à la fois désirée et redoutée. L'orientation actuelle consiste à faire l'élevage en lignée pure dans un troupeau qui supportera les pertes et qui fournira aux Centres d'Insémination à bon compte de la semence pour des croisements industriels (VISSAC, I972).

\section{3. - Limite d'emploi}

En l'absence d'une recherche systématique et en raison même de leur rareté et du temps exigé par leur étude les gènes utilisables individuellement en sélection ne peuvent remplacer la sélection quantitative. Il ne peut être question le lâcher la proie pour l'ombre. Simplement, toutes les fois qu'un d'entre eux se présentera il faudra lui trouver un mode d'emploi optimum dans le contexte sélectif du moment.

\section{LE FUTUR}

Il est certain que quelques-unes des propositions du récent article collectif consacré à la génétique humaine par la revue Time (ANONYME, I97I) sont des boutades si l'on veut les appliquer à notre espèce.

Par contre, les techniques permettant 1'abandon de la reproduction sexuée pour des espèces comme les Bovins où l'entretien des femelles mères des veaux à viande est d'un coût élevé feraient faire un bond énorme à la productivité.

De même la perspective de clonage de certains individus particulièrement bien adaptés à telle ou telle production n'est pas à considérer à la légère.

De telles techniques donneraient sans doute encore une meilleure chance d'utilisation aux mutants individuels tels que nous les avons étudiés. Mais, surtout, on nous promet certaines facilités pour modifier à volonté et avec précision les séquences d'ADN, c'est-à-dire les gènes eux-mêmes. 
A côté des applications médicales de telles techniques ressortissant d'une sorte de " génie génétique ", il y aura certainement pour les animaux domestiques des applications qui n'auront pas, comme chez l'Homme, à tenir compte des barrières morales.

\section{CONCLUSION}

Comme tous les progrès techniques l'utilisation des gènes individuels en sélection a suivi des chemins tortueux dont certains remontent très loin dans le passé. Son intérêt est apparu très lentement et de nombreuses personnes ont contribué consciemment et inconsciemment à son avènement.

Cette technique est encore d'un emploi limité mais elle a fait ses preuves. Elle mérite certainement d'être développée, aidant et relayant la sélection quantitative classique.

Rę̧u pour publication en octobre 1971.

\section{SUMMARY}

\section{CONSCIOUS AND UNCONSCIOUS UTILIZATION OF SOME HEREDITARY FACTORS IN SELEC'TING BIGGER DOMESTIC ANIMAL, SPECIES}

Recent studies have shown that most ordinary segregation mutations in domestic species do not have qualities such that the selectioner should give them individual attention. Thus, the important place of quantitative genetics in selection may be justified.

However, there is a class of genes having both favorable and unfavorable pleiotropic effects which may greatly improve the quality of animals being raised for production. In the past, they were used at the insistence of breeders, and that explains some of the differences between domestic and wild breeds before rational selection was employed.

Since the success of dwarf fowl selection, they arouse some interest in large mammals as is shown in the great amount of work being done on the bovine double-muscle character.

The common rule for their application is to minimize their negative and to emphasize their positive effects, depending on the type of gene. The latter may lead to a variety of utilizations in pure bred or in crossing.

Such "good genes" are also rather rare and at present we cannot count on them only for setting-up selection programs. However, with the progress of "genetic engineering" which allows us to create new mutations at will, the situation may be reversed.

\section{ZUSAMMENFASSUNG}

\section{BEWUSSTE UND UNBEWUSSTE AUSNUTZUNG VON ERBFAKTOREN IN DER SELEKTION DER GRÖSSEREN HAUSTIERARTEN}

Jüngste Untersuchungen haben gezeigt, dass die meisten Mutanten im gewöhnlichen Genabsonderungsprozess der Haustiere keine solche Vorrangstellung einnehmen, dass sie individuell für eine Selektion ausschlaggebend wären. Dadurch wirk im gewissen Sinne die Bedeutung gerechtfertigt, die der quantitativen genetischen Selektion beigemessen wird.

Es gibt jedoch eine Gen-Kategorie mit doppelter (sowohl negativer wie positiver), pleiotropischer Auswirkung, die die Rendite der gehaltenen Tiere bedeutend verbessern kann. In der 
Vergangenheit haben Züchter, ohne sich dessen bewusst zu sein, bereits diese Gene ausgenutzt, wodurch erklärt wird, warum es auch vor der Einführung der zielbewussten Selektion gewisse Unterschiede zwischen den Haustier- und den wilden Tierrassen gab. Auf Grund des Erfolges bei der Zwerghühnerselektion erwecken diese Gene heute auch ein gewisses Interesse bei den grossen Haustieren, wie die Fülle der Arbeiten über die Eigenschaft der muskulären Hypertrophie (Doppellender) bei den Rindern beweist.

Als Faustregel für ihre Verwendung gilt : Verkleinerung der dabei auftretenden Nachteile und Vergrösserung der guten Eigenschaften, was je nach Genart zu einer vielseitigen Nutzung sowohl in der direkten Abstammung als auch bei Kreuzungen führen kann.

Dermassen "gute Gene " sind aber ziemlich selten. Daher ist man im Augenblick noch weit davon entfernt, auf sie allein abzustellen bei der Aufstellung von Selektionsprogrammen. Immerhin versprechen die Fortschritte, die das " genetische Ingenieurwesen " erreicht, eine Verbesserung der gegenwärtigen Situation durch das Erschaffen nach Belieben von neuen Mutanten.

\section{RÉFÉRENCES BIBLIOGRAPHIQUES}

Anonyme, I97I. Man into superman. The promise and peril of the new Genetics, Time, apr. I9, I971 $25-44$.

Buschmann M., Schmid D. O., I968. Serumgruppen bei Tieren. Paul Parey, Berlin 54-58.

Hanser R., I965. Recherches sur la White Heifer Disease et son déterminisme génétique. I. R. S. I. A. Bruxelles. $C . R$. de recherches $\mathrm{n}^{\circ} 33,177 \mathrm{p}$.

Hrll W. G., I97I. Theoretical aspects of cross breeding. Ann. Génét. Sél. anim. 3, 23-34.

Lauvergne J.-J., I968. Catalogue des anomalies héréditaires des bovins (Bos taurus L.). Bull. tech. Dépt Genét. anim (Inst. nat. Rech. agron. Fr.) n ${ }^{\circ} \mathrm{I}, 9 \mathrm{I}$ p.

Lauvergne J.-J., I969. Progrès des connaissances génétiques sur l'intersexualité associée à l'absence de cornes chez la chèvre d'origine alpine. Ann. Génét. Sél. anim. 1, 403-4I2.

Lauvergne J.-J., I970. Gonadal hypoplasia and white color in Swedish Highland cattle. J. Hered. 61, 43-44.

Lauvergne J.-J., Boyazoglu H.-G., Denise Hubert, x968. Le phénomène culard chez les bovins, bibliographie annotée. Bull. tech. Dépt. Génét. anim. (Inst. Natn Rech. Agron. Fr.) nº 2, 49 p.

Lush J.-L., I965. Dairy cattle Genetics. J. Dairy Sci., 39, 693-694.

Mérat P., r970. Mendelian genetics and selection for quantitative traits in poultry: result and perspectives. World's Poult. Sci. J., 26, 57 r-586.

Mérat P. I972. Utilisation de facteurs mendéliens en élevage : l'exemple de la Poule. Ann. Génét. Sél. anim., 4,

Mérat P., Perramon A., I968. Groupes sanguins et productivité chez les volailles. World's Rev. Anim. Prod., 3, I3, 84-89.

Nordskog A. W., I964. Poultry immunogenetics. World's Poult. Sci. J., 20, I83-192.

ReNDEL J. M., I952. White heifer disease in a herd of dairy Shorthorn. J. Genet., 51, 89-94.

Robertson A., I 961 . Reviews of the progress of dairy science, Section A. Physiology. Genetics of Dairy cattle. J. Dairy Res., 28, 195-207.

RoBertson A., I966. Biochemical polymorphisms in animal improvement. Ve Congrès Europ. Groupes sang. polymorph. bioch. anim., Paris, 35-42.

Robertson A., I970. Molecular biology and animal breeding. Ann. Génét. Sél. anim. 2, 393-402.

Vissac B., I972. L'hypertrophie musculaire d'origine génétique ou caractère culard. Ann. Génét. Sél. anim., 4, 